\title{
The structure of schizotypal personality traits: a cross-national study
}

\author{
E. Fonseca-Pedrero ${ }^{1,2 *}$, M. Debbané ${ }^{3,4}$, J. Ortuño-Sierra ${ }^{1}$, R. C. K. Chan ${ }^{5}$, D. C. Cicero ${ }^{6}$, L. C. Zhang $^{7}$, \\ C. Brenner ${ }^{7}$, E. Barkus ${ }^{8}$, R. J. Linscott ${ }^{9}$, T. Kwapil ${ }^{10}$, N. Barrantes-Vidal ${ }^{11}$, A. Cohen ${ }^{12}$, A. Raine ${ }^{13}$, \\ M. T. Compton ${ }^{14}$, E. B. Tone ${ }^{15}$, J. Suhr ${ }^{16}$, J. Muñiz ${ }^{2,17}$, A. Fumero ${ }^{18}$, S. Giakoumaki ${ }^{19}$, I. Tsaousis ${ }^{19}$, \\ A. Preti ${ }^{20}$, M. Chmielewski ${ }^{21}$, J. Laloyaux ${ }^{22,23,24}$, A. Mechri ${ }^{25}$, M. A. Lahmar ${ }^{25}$, V. Wuthrich ${ }^{26}$, \\ F. Larøi ${ }^{22,23,24}$, J. C. Badcock ${ }^{27}$ and A. Jablensky ${ }^{28}$
}

${ }^{1}$ Department of Educational Sciences, University of La Rioja, Logroño, Spain; ${ }^{2}$ Center for Biomedical Research in the Mental Health Network (CIBERSAM), Oviedo, Spain; ${ }^{3}$ Faculty of Psychology and Educational Sciences, University of Geneva, Geneva, Switzerland; ${ }^{4}$ Department of Clinical, Educational and Health Psychology, University College London, London, UK; ${ }^{5}$ Neuropsychology and Applied Cognitive Neuroscience Laboratory, CAS key Laboratory of Mental Health, Beijing, China; ${ }^{6}$ Department of Psychology, University of Hawaii at Manoa, Honolulu, HI, USA; ${ }^{7}$ Department of Psychology, University of British Columbia, Vancouver, BC, Canada; ${ }^{8}$ School of Psychology, University of Wollongong, Wollongong, Australia; ${ }^{9}$ Department of Psychology, University of Otago, Dunedin, New Zealand; ${ }^{10}$ Department of Psychology, University of North Carolina at Greensboro, Greensboro, NC, USA; ${ }^{11}$ Department of Clinical and Health Psychology, Universitat Autònoma de Barcelona, Barcelona, Spain; ${ }^{12}$ Department of Psychology, Louisiana State University, Louisiana, LA, USA; ${ }^{13}$ Departments of Criminology, Psychiatry and Psychology, University of Pennsylvania, Philadelphia, PA, USA; ${ }^{14}$ Department of Psychiatry, Lenox Hill Hospital, New York, NY, USA; ${ }^{15}$ Department of Psychology, Georgia State University, Atlanta, GA, USA; ${ }^{16}$ Department of Psychology, Ohio University, Athens, OH, USA; ${ }^{17}$ Department of Psychology, University of Oviedo, Oviedo, Spain; ${ }^{18}$ Department of Psychology, University of La Laguna, Santa Cruz de Tenerife, Spain; ${ }^{19}$ Department of Psychology, University of Crete, Rethymno, Greece; ${ }^{20}$ Genneruxi Medical Center, Cagliari, Italy; ${ }^{21}$ Department of Psychology, Southern Methodist University, Dallas, TX, USA; ${ }^{22}$ Department of Biological and Medical Psychology, University of Bergen, Bergen, Norway; ${ }^{23}$ NORMENT - Norwegian Center of Excellence for Mental Disorders Research, University of Oslo, Oslo, Norway; ${ }^{24}$ Psychology and Neuroscience of Cognition Research Unit, University of Liège, Liège, Belgium; ${ }^{25}$ Psychiatry Department, University Hospital of Monastir, Monastir, Tunisia; ${ }^{26}$ Centre for Emotional Health, Department of Psychology, Macquarie University, Sydney, Australia; ${ }^{27}$ Centre for Clinical Research in Neuropsychiatry, Division of Psychiatry, Faculty of Health and Medical Sciences, University of Western Australia, Perth, Australia; ${ }^{28}$ Centre for Clinical Research in Neuropsychiatry, School of Psychiatry and Clinical Neurosciences, University of Western Australia, Perth, Australia

Background. Schizotypal traits are considered a phenotypic-indicator of schizotypy, a latent personality organization reflecting a putative liability for psychosis. To date, no previous study has examined the comparability of factorial structures across samples originating from different countries and cultures. The main goal was to evaluate the factorial structure and reliability of the Schizotypal Personality Questionnaire (SPQ) scores by amalgamating data from studies conducted in 12 countries and across 21 sites.

Method. The overall sample consisted of 27001 participants ( $37.5 \%$ males, $n=4251$ drawn from the general population). The mean age was 22.12 years (S.D. $=6.28$, range $16-55$ years). The SPQ was used. Confirmatory factor analysis (CFA) and Multilevel CFA (ML-CFA) were used to evaluate the factor structure underlying the SPQ scores.

Results. At the SPQ item level, the nine factor and second-order factor models showed adequate goodness-of-fit. At the SPQ subscale level, three- and four-factor models displayed better goodness-of-fit indices than other CFA models. MLCFA showed that the intraclass correlation coefficients values were lower than 0.106 . The three-factor model showed adequate goodness of fit indices in multilevel analysis. The ordinal $\alpha$ coefficients were high, ranging from 0.73 to 0.94 across individual samples, and from 0.84 to 0.91 for the combined sample.

Conclusions. The results are consistent with the conceptual notion that schizotypal personality is a multifaceted construct and support the validity and utility of SPQ in cross-cultural research. We discuss theoretical and clinical implications of our results for diagnostic systems, psychosis models and cross-national mental health strategies.

Received 14 February 2017; Revised 9 May 2017; Accepted 7 June 2017

Key words: Factorial validity, Psychosis risk, psychosis, schizotypal personality, schizotypy, SPQ.

\footnotetext{
* Address for correspondence: E. Fonseca-Pedrero, University of La Rioja, C/Luis de Ulloa, s/n, Edificio VIVES; C.P: 26002, Logroño, La Rioja, Spain.

(Email: eduardo.fonseca@unirioja.es)
}

\section{Introduction}

Schizotypal traits are considered a phenotypic-indicator of schizotypy, a latent personality organization reflecting a putative liability for schizophrenia-spectrum disorders (Meehl, 1962). These traits refer to anomalies 
across cognitive (e.g., hallucinations, ideas of reference), social/emotional (e.g., constricted affect, no close friends) and behavioural (e.g., odd behaviour and language) systems, that do not meet clinical threshold for psychotic disorders (Raine, 2006; Kwapil \& Barrantes-Vidal, 2015). Recent conceptualizations of the schizotypy framework indicate that it provides a unifying construct that efficiently links a broad continuum of clinical and subclinical psychosis manifestations, as well as normal personality variation (Kwapil \& Barrantes-Vidal, 2015). Understanding schizotypal traits in non-clinical samples may help elucidate aetiological mechanisms, provide a window to examine risk and protective factors without certain confounding factors (e.g., medication), and provide a necessary step in the process of developing early detection strategies and preventive interventions for those individuals at risk for psychosis-spectrum disorders (Barrantes-Vidal et al. 2015).

Previous research has shown that schizotypal traits are a valid putative phenotypic indicator for psychosisspectrum disorders (e.g., Lenzenweger, 2010; Fonseca Pedrero \& Debbané, 2017). First, considerable evidence from family, adoption and twin studies have demonstrated that schizotypal traits are related to schizophrenia (Kendler et al. 1993; Walter et al. 2016). Second, independent follow-up studies have shown that individuals from the general population and those at clinical or genetic high risk for psychosis who report schizotypal traits, as well as patients with schizotypal personality disorder, are at elevated risk for transition to psychosis and related conditions (Debbané et al. 2015). Third, schizotypal traits are qualitatively similar, but less severe than the symptoms found in patients with schizophrenia-spectrum disorders and ultra-high risk samples. In fact, schizotypal traits have been associated with similar deficits in brain function, eye movements, neurocognition, language, etc., amongst others, to those seen in patients with psychosis (Raine, 2006; Fusar-Poli et al. 2014; Cohen et al. 2015; Ettinger et al. 2015). Fourth, they share many of the same demographic and environmental concomitants as those found in patients with psychosis (e.g., trauma, cannabis use, high levels of urbanicity) (Linscott \& van Os, 2013). Fifth, isolated schizotypal traits, even those insufficient in severity or impairment to warrant a clinical diagnosis, are associated with increased risk of psychiatric morbidity (e.g., suicidal behaviour, mental health problems, low quality of life) and functional disability (Nuevo et al. 2012; Kwapil et al. 2013; Kelleher et al. 2014). For example, adolescents in a schizophrenia liability class - those who reported schizotypal traits showed greater odds of passive suicidal ideation at a 2-year follow-up compared with those not in the liability class [odds ratio (OR) 8.15, 95\% confidence interval
(CI) 1.34-49.60] (Schimanski et al. 2017). These findings reveal an important overlap in schizotypal traits and psychosis-spectrum disorders, supporting the notion of a phenomenological and etiological continuity.

To assess schizotypal traits, several tools have been developed. These instruments permit examination of variations in healthy trait schizotypy, as well as in the latent vulnerability to psychosis-spectrum disorders (e.g., Mason, 2015; Fonseca-Pedrero et al. 2016b). This psychometric high-risk methodology has shown validity and clinical relevance, in line with conventional interview-based high-risk approaches for psychosis (Barrantes-Vidal et al. 2013; Cicero et al. 2014). Moreover, self-report can be more sensitive to environmental $v$. heritable effects than to interview-based assessment (Kendler et al. 2007).

The Schizotypal Personality Questionnaire (SPQ) (Raine, 1991) is a popular, extensively used self-report tool for the assessment of schizotypal traits in both clinical and non-clinical populations. The SPQ measures a broad range of schizotypal traits - originally it encompassed nine subordinate traits that are based on the operational definition of DSM-III-R Schizotypal Personality Disorder (SPD) [American Psychiatric Association (APA), 1987]. These features also represent the main features of DSM-5 SPD criteria in the chapter on Schizophrenia spectrum and other psychotic disorders (APA, 2013). Notably, the DSM-5 also presents SPD in the context of an alternative hybrid (dimensional/categorical) model of personality disorders that is outlined in Section III (APA, 2013). The 74 items of the SPQ are distributed across nine subscales, each containing seven to nine items; these subscales encompass odd beliefs or magical thinking, unusual perceptual experiences, ideas of reference, paranoid ideation/suspiciousness, excessive social anxiety, no close friends, constricted affect, odd or eccentric behaviour and odd speech. The psychometric properties have been examined in a number of nation- or region-specific studies (e.g., Fonseca-Pedrero et al. 2014; Gross et al. 2014; Tsaousis et al. 2015; Cicero, 2016).

Although there is no universal agreement on the latent structure of schizotypy or psychosis liability, whether it is dimensional or categorical (Everett \& Linscott, 2015), the literature consistently holds that the phenotypic expression of schizotypal traits is multifaceted (e.g., Vollema \& Hoijtink, 2000; Fonseca-Pedrero et al. 2014; Gross et al. 2014; Tsaousis et al. 2015; Cicero, 2016). This multifaceted nature can be understood in terms of a latent multidimensional or factor structure framework. Using the SPQ (Raine, 1991), or its brief version (SPQ-B) (Raine \& Benishay, 1995), the threefactor model proposed by Raine et al. (1994), which comprises Cognitive-Perceptual (Positive), Interpersonal (Negative) and Disorganized dimensions, has been 
one of the most widely replicated models (Chen et al. 1997; Vollema \& Hoijtink, 2000; Fossati et al. 2003; Badcock \& Dragovic, 2006; Raine, 2006; Wuthrich \& Bates, 2006; Bora \& Arabaci, 2009; Fonseca-Pedrero et al. 2014, 2016a). To a large extent, this factorial structure of schizotypal personality is similar to that for clinical symptoms reported by patients with schizophrenia (Liddle, 1987). The four-factor model proposed by Stefanis et al. (2004) that includes Cognitive-Perceptual, Interpersonal, Disorganization, and Paranoid dimensions has also been frequently replicated (Bora \& Arabaci, 2009; Compton et al. 2009; Fonseca-Pedrero et al. 2014; Gross et al. 2014). In several studies, the goodness-of-fit indices reported in for Stefanis et al.'s model are similar to, or at times better, than those reported for Raine's model. Item-level examinations of the SPQ have yielded more complex factor solutions (Chmielewski \& Watson, 2008; FonsecaPedrero et al. 2014), suggesting a need for further replication and deeper analyses.

Variability across studies in the factorial composition of the SPQ may be due in part to the type and size of participant samples as well as the analysis methodologies that were employed. Also, it is noteworthy that different measurement models (e.g., bifactor model) or methodologies (e.g., Multilevel Confirmatory factor analyses - ML-CFA) may better capture the complexity and heterogeneity of schizotypal phenotype, as assessed by the SPQ, when comparing data from multiple countries. These measurement and methodological approaches, however, are not often applied in research on schizotypal traits and the extended psychosis phenotype.

As of yet, there has been no in-depth examination of the factorial structure underlying schizotypal traits, as measured with the SPQ that compares data from diverse countries and cultures. Moreover, no previous studies have examined whether the phenotypic expression of schizotypal traits is similar across sites or countries. It is often assumed that the structure of the schizotypal personality at the individual level is universal; however, this assumption has yet to be assessed empirically using data drawn from different geographical regions.

Therefore, in order to address these possible sources of inconsistency in prior research findings, our aim was to evaluate the factorial structure and reliability of the SPQ scores by amalgamating data from studies conducted in 12 countries and across 21 sites. In particular, the present study: (a) examined associations among self-reported schizotypal traits; (b) tested the factorial structure, at both item and subscale levels, of SPQ scores within and between samples; and (c) estimated the reliability of self-reported schizotypal traits. In line with previous evidence, we hypothesized that three- and four-factor models of the SPQ scores would provide the best fit to the data. Moreover, we further hypothesized that these measurement models of schizotypal personality would fit well in the multilevel analyses.

\section{Method \\ Participants}

This study was undertaken as part of the activities of the 1st International Consortium on Schizotypy Research celebrated in Geneva in 2014 (Debbané \& Mohr, 2015). Although this is not a meta-analysis, studies using the SPQ in the healthy adult population samples were identified by systematically searching Medline (PubMed and Ovid), PsycINFO, SCOPUS and ISI (Science and Social Science Citation Index) databases between June and August of 2014.

Citations in identified articles were also searched for additional sources. Access to data was sought for studies that met the following inclusion criteria: (a) the sample size was $\geqslant 100$; (b) the sample was obtained from the general population, including college or undergraduate populations (samples of non-clinical adolescents, school pupils, patients, or family members of patients with psychosis were excluded); (c) in the case of articles with possible overlapping samples, the study with a larger or more informative sample was selected; and (d) item-level data on the 74 SPQ items and information on the administration procedure (paper-pencil $v$. computerized) was available.

Table 1 provides a summary of the demographic characteristics of samples that were obtained (See eTable 1 online Supplementary Material). Item level data were obtained from 21 sites across 12 countries (USA, UK, China, Belgium, Spain, Italy, Tunisia, Australia, New Zealand, Canada, Mauritius and Greece). The overall sample consisted of 27001 participants ( $n=4251$ drawn from the general population). The mean age was 22.12 years (S.D. $=6.28$; range $16-$ 55 years), $15.2 \%(n=4113)$ of participants did not provide age. Only $3.3 \%(n=849)$ of the sample were aged over 35 years. Of participants, $37.5 \%(n=10126)$ were male, $60.6 \%(n=16368)$ were female and $1.9 \%(n=$ $507)$ did not specify gender.

Studies were reviewed and approved by institutional review boards or ethics committees of the jurisdictions in which studies were undertaken. All participants provided written informed consent prior to participation. Studies were conducted in accordance with the guidelines of the Declaration of Helsinki (World Medical Association, 2013). Except for five studies, the data used in the present study were published elsewhere. We deleted from the initial sample 
Table 1. Demographic characteristics of the sample

\begin{tabular}{|c|c|c|c|c|c|c|c|c|c|}
\hline Study & Country & Main researcher & $n$ & $\begin{array}{l}\text { Sampling/ } \\
\text { procedure }\end{array}$ & $\begin{array}{l}\text { Mean } \\
\text { age (S.D.) }\end{array}$ & $\begin{array}{l}\text { Age } \\
\text { range }\end{array}$ & $\begin{array}{l}\text { Males } \\
n(\%)\end{array}$ & $\begin{array}{l}\text { Missing } \\
\text { values, } \\
\text { age } n(\%)\end{array}$ & $\begin{array}{l}\text { Missing values, } \\
\text { gender } n(\%)\end{array}$ \\
\hline 1 & USA & Cicero & 3162 & College & $20(3.7)$ & $16-55$ & 997 (31.5) & $39(1.2)$ & $24(0.8)$ \\
\hline 2 & USA & Kwapil & 1556 & College & $19.5(2.9)$ & $16-54$ & $363(23.3)$ & $548(35.2)$ & $5(0.3)$ \\
\hline 3 & Spain & Fonseca-Pedrero & 1123 & College & $20.2(2)$ & $18-29$ & $224(19.9)$ & - & - \\
\hline 4 & USA & Compton & 1190 & College & $20.9(4)$ & $16-52$ & $284(23.9)$ & - & - \\
\hline 5 & USA & Chmielewski & 556 & College & - & - & $102(18.3)$ & $556(100)$ & - \\
\hline 6 & Mauritius & Raine & 1201 & Birth cohort & $23.4(1.2)$ & $21-27$ & $688(57.3)$ & $1(0.1)$ & - \\
\hline 7 & Italian & Preti & 649 & College & $24.3(3.5)$ & $19-38$ & $305(47)$ & - & - \\
\hline 8 & Australia & Wuthrich & 445 & College & $22.6(6.3)$ & $17-53$ & $126(28.3)$ & $8(1.8)$ & $3(0.7)$ \\
\hline 9 & USA & Cohen & 1458 & College & $19.3(2.2)$ & $16-53$ & $531(36.4)$ & $1(0.1)$ & - \\
\hline 10 & Belgium & Larøi & 357 & General & $25(10.3)$ & $17-55$ & $110(38.8)$ & - & - \\
\hline 11 & Australia & Badcock & 342 & General & 36.1 (11.6) & $17-55$ & $182(53.2)$ & $1(0.3)$ & - \\
\hline 12 & Belgium & Laloyaux & 536 & General & $24.9(8.1)$ & $18-55$ & $135(25.2)$ & - & - \\
\hline 13 & Tunisia & Mechri & 458 & College & $20.4(1.4)$ & $18-29$ & 137 (29.9) & - & - \\
\hline 14 & $\begin{array}{l}\text { New } \\
\text { Zealand }\end{array}$ & Linscott & 1648 & College & $20.1(3.1)$ & $17-51$ & $515(30.3)$ & - & - \\
\hline 15 & UK & Barkus & 774 & General & $21.6(4.4)$ & $17-49$ & $291(37.6)$ & - & - \\
\hline 16 & Australia & Barkus & 1144 & College & - & - & $326(28.5)$ & $1144(100)$ & - \\
\hline 17 & USA & Suhr & 1169 & College & - & - & $299(27.3)$ & 1169 (100) & 74 (6.3) \\
\hline 18 & China & Chan & 4907 & College & $19.7(1.6)$ & $16-24$ & $2973(60.6)$ & 644 (13.1) & $401(8.2)$ \\
\hline 19 & Canada & Zhang & 1849 & College & $20.8(2.9)$ & $18-53$ & $562(30.4)$ & - & - \\
\hline 20 & USA & Zhang & 1386 & MTurk & 31.9 (9.5) & $18-55$ & $586(42.3)$ & $1(0.01)$ & - \\
\hline 21 & Greece & Tsaousis & 1041 & General & $32.4(9.9)$ & $18-55$ & $390(37.5)$ & $1(0.01)$ & - \\
\hline
\end{tabular}

those participants with missing values for more than two SPQ items. Based on the SPSS missing value analysis module, the relatively few missing values in the data were replaced by regression-based estimates to which an error component was added.

\section{Instrument}

The SPQ (Raine, 1991) provided a common index of schizotypal traits across all study sites. Although designed for SPD as defined in the DSM-III-R (APA, 1987), the SPQ is still consistent with the DSM-5 (APA, 2013) because the nine symptoms have not changed (Cicero, 2016). In five studies, the SPQ was administered using a computerized format (studies: $4,8,12,19$ and 20) and in two studies a Likert response format (1-5) was used (studies: 8 and 9). For these two studies, the responses were recoded as ' $1-3$ ' to ' 0 ' (No) and ' $4-5$ ' to ' 1 ' (Yes). This data modification produces dichotomous response frequencies consistent with scores from the original SPQ. In the present work we used the SPQ versions adapted and validated for each country: English version (Raine, 1991), Spanish (Fonseca-Pedrero et al. 2014), Italian (Fossati et al. 2003), Chinese (Chen et al. 1997), Arabic (Lahmar et al. 2014), French (Dumas et al. 2000), Creole (Reynolds et al. 2000) and Greek (Tsaousis et al. 2015).

\section{Data analyses}

Several analyses were carried out in the present study. First, descriptive statistics and correlations between SPQ subscales were computed.

Second, given the hierarchical structure of the data, with participants nested in sites/countries, a ML-CFA was performed. ML-CFA decomposes the total variance into two components (i.e., within-site variance and between-site variance). Therefore, this approach allows researchers to construct measurement models at both individual and country levels (i.e., within-level and between-level) (e.g., Cheung et al. 2006; Byrne, 2012). Prior to conducting the ML-CFA, two steps were performed: a) multiple CFA models were tested on the total sample as well as at site level; and b) once we determined the best-fitting measurement model, intraclass correlation coefficients (ICCs) were estimated. The ICC assesses the level of variance in an observed variable that is attributable to membership in its cluster (e.g., site). ICC values range from 0.0 to 1.0. A high ICC implies that the betweengroup variance dominates the within-group variance. Previously published studies have reported that the presence of ICCs that exceed 0.10 warrants the use of ML-CFA (e.g., Cheung et al. 2006; Byrne, 2012). These two steps provided initial information about the factor 
structure of the SPQ, as well as pertinent information used to justify multilevel analyses. Finally, with the best fitting measurement models, two-level CFAs with continuous factor indicators were conducted.

Several measurement models were tested at both item and subscale level. At the item-level, we tested four different factor models by means of CFAs. As SPQ items were binary, we used the weighted least squares means and variance adjusted (WLSMV) estimator (Muthén \& Muthén, 1998-2012). Model 1 was a one-factor latent structure; all 74 items loaded on a single factor. Model 2 was a nine-factor oblique structure where the nine factors corresponded to the nine SPQ subscales. Model 3 was a bifactor model with one general factor and nine specific factors (i.e., nine SPQ subscales). Model 4 was a second-order model, that is, one involving a hierarchical structure. Here, three second-order factors (corresponding to cognitiveperceptual, interpersonal and disorganization dimensions) loaded on nine lower-order factors. In this model paranoia items were allowed to saturate in both cognitive-perceptual and interpersonal factors, consistent with previous research (Raine et al. 1994). At the subscale level, we tested six models using an MLM estimator. In Model 5, a single factor loaded onto the nine SPQ subscales (e.g., baseline model). Model 6 comprised two correlated factors (i.e., cognitive-perceptual and Interpersonal) (Gross et al. 2014). Model 7 comprised three correlated factors (i.e., cognitive-perceptual, interpersonal and disorganized). Model 8 was a variation of Model 7 in which the positive and interpersonal factors both loaded on paranoid ideation (Raine et al. 1994). Model 9 was a four-factor model based on Stefanis et al. (2004) (i.e., cognitive-perceptual, paranoid, interpersonal and disorganized). Model 10 was a subscale-level bifactor model with a general schizotypal factor and three specific factors corresponding to cognitive-perceptual, interpersonal and disorganized dimensions.

The goodness-of-fit indices employed were: $\chi^{2}$, the Comparative Fit Index (CFI), the Tucker-Lewis index (TLI), the Root Mean square Error of Approximation (RMSEA) (and 90\% CI), the Weighted Root Mean Square Residual (WRMR) for dichotomous indicators, the Standardized Root Mean Square Residual (SRMR) for continuous indicators, and the Akaike Information Criterion (AIC) and Bayesian Information Criterion (BIC). Good fit is indicated when the CFI and TLI are over 0.95 and the RMSEA is under 0.08 (reasonable fit) or under 0.05 (good fit) (Hu \& Bentler, 1999; Brown, 2015). The presence of WRMR values below 1.0 has been suggested as indicative of adequate model fit ( $\mathrm{Yu}$ \& Muthén, 2002). The AIC and BIC do not have cut-off values. Instead, models with smaller AIC and BIC values have better fit. The AIC and BIC are useful because they penalize more complex models.

Finally, we calculated the internal consistency of the SPQ scores in each country as well as in the total sample, using ordinal $\alpha$ coefficients (Zumbo et al. 2007). Ordinal $\alpha$ performs well for analysis of dichotomous data and overcomes several problems associated with Cronbach's $\alpha$ (e.g., Dunn et al. 2014).

SPSS 22.0 (IBM Corp Released, 2013), Mplus 7.0 (Muthén \& Muthén, 1998-2012) and R (R Development Core Team, 2011) were used for data analyses.

\section{Results}

\section{Descriptive statistics and Pearson correlations between schizotypal traits}

Descriptive statistics of the SPQ subscales are reported in eTable 2 (online Supplementary Material). Table 2 shows the mean (and range) of Pearson's correlations among the schizotypal subscales across studies. According to convention, correlations of $0.1,0.3$ and 0.5 are regarded as small, medium and large in effect size, respectively (Cohen, 1988). There were several notable findings. First, the magical thinking subscale was relatively independent of the Odd behaviour, Odd speech and No close friends subscales. In fact, these associations were the only ones that were not statistically significant and close to zero. Second, the correlations among the remaining SPQ subscales were of medium to large effect size. No subscales were redundant (i.e., with $r>0.85$ ). Third, the Odd Speech subscale was highly correlated with No Close Friends. Fourth, Excessive Social Anxiety was highly correlated with the Ideas of Reference and Unusual Perceptual Experiences subscales.

\section{Structure of schizotypal traits: CFA for the full sample and across samples}

Table 3 presents the goodness-of-fit indices for the models tested for the full sample. At the item level, we selected the RMSEA as the primary index of model fit because it has been generally identified as the best performing index for the WLSMV method. RMSEA values of less than 0.06 reliably indicate good model fit for binary outcomes (Yu \& Muthén, 2002). Thus, at the item level, the measurement models that displayed the best goodness-of-fit indices were Model 3 (the nine-first-order model) and Model 4 (the three-second-order model).

At the subscale level, the CFA models that showed the best fit were Raine et al.'s model with positive and interpersonal factors both loading on Paranoid ideation, and Stefanis et al.'s four-factor model. In particular, Stefanis 
et al.'s model yielded better goodness-of-fit indices than the competing factorial models.

eTable 3 (online Supplementary Material) shows the goodness-of-fit indices for the CFA models tested for each subsample. In all sites, the goodness-of-fit indices for the Stefanis et al. (2004) model met good fit criteria. Other factor models tested, such as the bifactor model and three-factor model of Raine et al. (1994), also showed acceptable fit.

The three and four-factor models tested at the subscale level fit the data well in the full sample and in all $21 \mathrm{sub}-$ samples. Based on previous models of schizotypal personality and higher goodness-of-fit indices, we retained the three and four-factor models, at the subscale level, as the models that best accounted for the factor loadings and associations among latent factors.

\section{The three- and four-factor models of schizotypal traits}

Table 4 shows the standardized loadings estimated for the total sample, as well as the ranges of standardized factor loadings for the four-factor model for each study. The Unusual Perceptual Experiences and Ideas of Reference subscales had the highest factor loadings across studies. Correlations among the four latent factors ranged from $0.31 \quad(0.10-0.51)$ for the paranoid-interpersonal factors to $0.68(0.59-0.79)$ for the positive-disorganization factors $(p<0.01)$.

Table 5 shows the standardized factor loadings for the total sample, as well as the ranges of standardized loadings estimated for the three-factor model for each study. Correlations among the three latent factors ranged from $0.39(0.14-0.60)$ for the positive-interpersonal factors to $0.74(0.62-0.82)$ for the positive-disorganized factors $(p<0.01)$.

\section{ML-CFA of the three-factor model}

Multilevel four-factor model estimation could not be completed due to a non-positive matrix; consequently, no results could be obtained for this measurement model. Thus, only the three-factor model was tested in multilevel analyses. For the three-factor model, all SPQ subscales showed ICC values lower than 0.106 , Thus, the amount of variance attributable to cluster membership (i.e., site) was lower than $11 \%$. ICC values were: Ideas of Reference $=0.097$; Unusual Perceptual Experiences $=0.059$; Magical Thinking $=0.086$; Paranoid Ideation $=0.106$; Excessive Social Anxiety $=0.035$; No Close Friends $=0.079$; Constricted Affect $=0.065$; Odd Speech $=0.056$; Odd Behaviour $=0.038$. These results indicates that a ML-CFA could be warranted; however, the hierarchical nature of the data did not have a clear significant effect on the factor structure of the SPQ (i.e., almost all ICCs values lower than 0.10 ). 
Table 3. Goodness-of-fit indices of the schizotypal personality models tested for the full sample $(n=27001)$

\begin{tabular}{|c|c|c|c|c|c|c|c|c|c|}
\hline Model & $\chi^{2}$ & $d f$ & RMSEA $(90 \% \mathrm{CI})$ & CFI & TLI & WRMR & SRMR & AIC & $\mathrm{BIC}$ \\
\hline \multicolumn{10}{|l|}{ Item level } \\
\hline 1-factor & 320933.11 & 2627 & $0.067(0.067-0.067)$ & 0.608 & 0.597 & 11.861 & - & - & - \\
\hline 9-factors & 130596.38 & 2591 & $0.043(0.043-0.043)$ & 0.842 & 0.836 & 7.250 & - & - & - \\
\hline Bifactor +9 factors & 194629.42 & 2553 & $0.053(0.053-0.053)$ & 0.763 & 0.750 & 8.967 & - & - & - \\
\hline 3 higher-order +9 factors & 140677.86 & 2614 & $0.033(0.033-0.033)$ & 0.830 & 0.824 & 7.720 & - & - & - \\
\hline \multicolumn{10}{|l|}{ Subscale level } \\
\hline 1-factor & 21911.59 & 27 & $0.173(0.171-0.175)$ & 0.733 & 0.644 & 19.167 & 0.094 & 978649.51 & 978871.01 \\
\hline 2-factors & 11623.01 & 26 & $0.129(0.127-0.131)$ & 0.859 & 0.804 & 15.249 & 0.073 & 966819.95 & 967049.66 \\
\hline 3-factors & 8775.08 & 24 & $0.116(0.114-0.118)$ & 0.893 & 0.840 & 14.342 & 0.069 & 963524.01 & 963770.12 \\
\hline 3-factors (overlap PI) & 5079.75 & 23 & $0.090(0.088-0.092)$ & 0.938 & 0.903 & 9.431 & 0.044 & 959342.69 & 959597.01 \\
\hline 4 -factors & 2337.16 & 23 & $0.067(0.065-0.070)$ & 0.972 & 0.946 & 6.492 & 0.030 & 956251.95 & 959539.07 \\
\hline Bifactor +3 factors & 5233.56 & 19 & $0.101(0.099-0.103)$ & 0.936 & 0.880 & 9.710 & 0.046 & 959531.59 & 959818.72 \\
\hline
\end{tabular}

PI, Paranoid Ideation; $\chi^{2}$, Chi Square; df, Degrees of freedom; RMSEA, Root Mean Square Error of Approximation; CI, Confidence Interval; CFI, Comparative Fit Index; TLI, Tucker-Lewis Index; SRMR, Standardized Root Mean Square Residual; WRMR, Standardized Root Mean Square Residual; AIC, Akaike Information Criterion; BIC, Bayesian Information Criterion.

The three-factor model of Raine et al. (1994) showed adequate goodness of fit indices $\left(\mathrm{S}-\mathrm{B} \chi^{2}=3021.22 ; \mathrm{df}=46\right.$; $\mathrm{CFI}=0.958 ; \mathrm{TLI}=0.935 ; \mathrm{RMSEA}=0.049 ; \mathrm{SRMR}_{\text {Within }}=$ $0.045 ; \mathrm{SRMR}_{\text {Between }}=0.128 ; \mathrm{AIC}=943269.12 ; \mathrm{BIC}=943$ 703.92). eTable 4 (online Supplementary Material) shows the standardized loadings estimated for the threefactor model tested in multilevel analyses. The standardized factor loadings were slightly higher at the site level.

\section{Reliability estimations of the schizotypal traits}

Table 6 shows the internal consistency values for the SPQ subscales across studies, as well as for the total sample. Ordinal $\alpha$ coefficients were high and ranged between 0.73 and 0.94 for the subscales in the individual samples, and from 0.84 to 0.91 for the total sample.

\section{Discussion}

The SPQ (Raine, 1991) is one of the most frequently used self-report tools for assessing schizotypal traits in samples of the general population as well as in clinical samples. Moreover, the SPQ may have utility as a screening instrument that can identify individuals who may be at increased risk for psychosis-spectrum disorders (Mason, 2015; Fonseca-Pedrero et al. 2016b). To date, there have been no comprehensive reports on the structure of the schizotypal personality using large and representative multi-national or multi-ethnic samples. We sought to bring clarity to this matter by examining the SPQ's factorial structure and reliability across different studies and countries. Notably, this is the first study to include data from multiple continents and is the largest SPQ dataset to be collated to date.
Such a cross-national investigation of the SPQ has potential to advance our understanding of the manifestation of schizotypal traits across the world. In addition, a multisite data set is helpful because it contributes to knowledge about the external validity and generalizability of schizotypal personality.

Examination of the factorial structure underlying the SPQ scores indicates that schizotypal traits have a multidimensional rather than a unidimensional structure. At the item level, the nine factor and second-order factor models had adequate goodness-of-fit (i.e., based on RMSEA indices), especially given the factorial complexity of these measurement models (i.e., 74 categorical items and nine first-order factors or three-higher order factors). Moreover, almost all factorial loadings were high and statistically significant across studies and countries. These results are consistent with the theoretical grouping of the nine SPQ subscales as well as with a three-factor model of schizotypal personality (Raine, 1991). To date, no previous studies have tested these CFA measurement models of the SPQ at the item level. In future studies, these findings need replication and deeper analysis; for example, it will be important to study measurement invariance of the SPQ items across countries.

At the subscale level, the three-factor model of Raine et al. (1994) and the four-factor model of Stefanis et al. (2004) were the best fitting across studies. First, the three-factor model in which the Paranoid subscale saturated on both positive and interpersonal dimensions, showed a better fit to the data for the full sample and across samples. Our findings converge with those obtained in studies that focus on abbreviated versions of the SPQ (Fonseca-Pedrero et al. 2009; Cohen et al. 
Table 4. Standardized factor loadings for the four-factor model

\begin{tabular}{|c|c|c|c|c|}
\hline & \multicolumn{4}{|l|}{ Factor } \\
\hline & I & II & III & IV \\
\hline MGT & $0.59(0.50-0.86)$ & & & \\
\hline UPE & $0.87(0.73-0.95)$ & & & \\
\hline IREF & & $0.93(0.79-0.99)$ & & \\
\hline ESA & & $0.15(0.01-0.29)$ & $0.57(0.43-0.64)$ & \\
\hline PI & & $0.43(0.39-0.59)$ & $0.42(0.29-0.44)$ & \\
\hline $\mathrm{NCF}$ & & & $0.81(0.69-0.85)$ & \\
\hline CA & & & $0.83(0.75-0.88)$ & \\
\hline OB & & & & $0.68(0.56-0.77)$ \\
\hline OS & & & & $0.77(0.41-0.86)$ \\
\hline
\end{tabular}

MGT, Magical Thinking; UPE, Unusual Perceptual Experiences; IREF, Ideas of Reference; ESA, Excessive Social Anxiety; PI, Paranoid Ideation; NCF, No Close Friends; CA, Constricted Affect; OB, Odd Behaviour; OS, Odd Speech.

Note. Brackets shows range values of the standardized factorial loadings estimated across 21 studies. All standardized factorial loadings estimated were statistically significant $(p<0.01)$.

Table 5. Standardized factorial loadings for the three-factor model in the total sample $(n=27001)$

\begin{tabular}{llll}
\hline & Factor & & \\
\cline { 2 - 4 } & I & II & III \\
\hline MGT & $0.73(0.34-0.68)$ & & \\
UPE & $0.58(0.50-0.82)$ & & \\
IREF & $0.78(0.62-0.82)$ & & \\
PI & $0.39(0.34-0.61)$ & $0.42(0.22-0.42)$ & \\
ESA & & $0.63(0.49-0.71)$ & \\
NCF & & $0.81(0.69-0.86)$ & \\
CA & & $0.83(0.75-0.88)$ & $0.68(0.54-0.77)$ \\
OB & & & $0.77(0.42-0.86)$ \\
OS & & &
\end{tabular}

MGT, Magical Thinking; UPE, Unusual Perceptual Experiences; IREF, Ideas of Reference; ESA, Excessive Social Anxiety, PI: Paranoid Ideation; NCF, No Close Friends; CA, Constricted Affect; OB, Odd Behaviour; OS, Odd Speech.

Note. Brackets shows range values of the standardized factorial loadings estimated across 21 studies. All standardized factorial loadings estimated were statistically significant $(p<0.01)$.

2010) as well as previous factorial studies in both clinical and non-clinical samples (Chen et al. 1997; Vollema \& Hoijtink, 2000; Fossati et al. 2003; Badcock \& Dragovic, 2006; Wuthrich \& Bates, 2006; Bora \& Arabaci, 2009; Fonseca-Pedrero et al. 2016a). Similar factorial solutions have been found for other measures of schizotypy in samples of the general population (Fonseca-Pedrero et al. 2015). Moreover, this factorial structure is to a large extent similar to that reported in studies of patients with psychosis (Liddle, 1987). Just as the manifestation of schizophrenia is heterogeneous - encompassing a broad range of emotional, cognitive, perceptual, social and behavioural functions - schizotypy also involves a diverse set of traits (Cohen \& Fonseca-Pedrero, in press).

Second, Stefanis et al.'s (2004) model yielded the best goodness-of-fit indices in comparison with the other measurement models. These results are convergent with those reported by previous researchers (e.g, Bora \& Arabaci, 2009). Our results show that the SPQ may be particularly useful for tapping positive, interpersonal, paranoid and disorganized schizotypal features. However, the results for the four-factor model should be interpreted cautiously. Specifically, in Stefanis et al.'s model the interpersonal and paranoid factors have two subscales in common - Excessive Social Anxiety and Paranoid Ideation. From a psychometric point of view, such cross-loading of subscales renders interpretation problematic. In particular, it becomes difficult to understand what each dimension measures. These limitations have to be taken into account when interpreting the significance of results within a CFA framework.

Third, correlated three-factor multilevel model with loading freely estimated across levels indicated good fit of the model to the data. This schizotypal measurement model seems to be similar at both individual and country levels. The findings presented in this study favour the use of the three-factor model of the SPQ, at least in the countries included in this study. In adition, our multilevel results provide new insight into the construct of schizotypal personality. However, more research is needed; it will be important to 
replicate these findings in samples drawn randomly from the general population, to test scalar and strong measurement invariance at multilevel data, and to add data from new countries. According to Cohen and Fonseca-Pedrero (in press) resolving the structure of schizotypal personality is an important step towards: (a) understanding the number and content of schizotypy symptoms, (b) resolving whether schizotypy reflects multiple processes or a single construct with varied expressions, and (c) developing more sophisticated measures and operational definitions for empirical and clinical use. Moreover, a sound and reliable factorial solution may harmonize clinical and empirical research on schizotypal traits worldwide.

The SPQ scores showed adequate levels of internal consistency across studies and countries. The reliability of the SPQ scores, estimated with ordinal $\alpha$, were above 0.75 . SPQ scores showed adequate psychometric properties across countries and hold implications for the use of this tool in cross-cultural research as well as for early detection of those individuals at risk for psychosis-spectrum disorders and mental health disorders. The SPQ is a tool that covers a wide variety of facets related to schizotypal personality, and, therefore, it can be considered as an accurate and useful tool to measure the wide scope of phenomena captured by this construct included within DSM-5 and ICD-10. Moreover, psychometric measurement of schizotypal personality allows us to understand the various manifestations of psychosis-spectrum risk. The psychometric assessment of schizotypal traits offers unique benefits - it is relatively inexpensive, non-invasive and useful for screening large samples of the general population. This research further extends the knowledge of the reliability of schizotypal traits, measured using the SPQ, in non-clinical samples from different countries.

The results of the present study should be interpreted in the light of the following limitations. First, the majority of the participants were college students and this fact may affect generalization of the results to other populations of interest. Counter-balancing these cautions, we note that findings from general population samples (Studies 10, 11, 12, 15 and 21), birth cohort (Study 6) and older samples (Studies 11, 20 and 21) yielded findings consistent with those from college samples. Second, the study is subject to the problems inherent to any research based on selfreports. This notwithstanding, self-report has the advantages that it is free of independent observer biases and can be more sensitive to underlying causal processes (Kendler et al. 2007). Third, the infrequency response was not systematically employed in all samples. Fourth, we have not considered whether the latent structure of the SPQ is best conceived as 
dimensional or categorial in nature. That is, our findings do not speak to whether we should think of the latent structure of schizotypal personality as comprising latent classes, latent dimensions, or some combination of dimensions and classes (Linscott, 2013).

\section{Conclusions}

Schizotypal personality is a heterogeneous construct closely linked to psychosis-spectrum disorders supported by an extensive body of theoretical and empirical knowledge. This study is the first to comprehensively examine the underlying structure and reliability of self-reported schizotypal traits using a multinational sample. First, the results strengthen the conceptual notion that schizotypal personality is a multifaceted rather than a unitary construct. Second, the SPQ, a tool that covers a wide variety of facets of schizotypal personality, showed adequate psychometric properties across countries. The current findings have important theoretical and clinical implications for psychosis-spectrum disorders, aetiological models and international diagnostic systems. Advances in the field of measurement open up new horizons for the assessment of schizotypal personality traits and allow a better understanding of the structure and content of this construct across western and non-western countries.

\section{Supplementary material}

The supplementary material for this article can be found at https://doi.org/10.1017/S0033291717001829

\section{Acknowledgements}

E.F.P was supported by the Spanish Ministry of Science and Innovation (MICINN) (PSI2014-56114-P), by the Instituto Carlos III, Center for Biomedical Research in the Mental Health Network (CIBERSAM), and by 2015 edition of the BBVA Foundation Grants for Researchers and Cultural Creators. M.D was supported by the Swiss National Science Foundation (100019_159440). R.Ch. was supported by the Beijing Training Project for Leading Talents in S\&T (Z151100000315020), the Beijing Municipal Science \& Technology Commission Grant (Z161100000216138), and the CAS/SAFEA International Partnership Programme for Creative Research Teams (Y2CX131003). S. G and I.T. were supported by the 'ARISTEIA II' Action of the Operational Programme Education and Lifelong Learning and was co-funded by the European Social Fund (ESF) and National Resources [grant number KA 2990].

\section{Declaration of Interest}

None.

\section{Ethical standards}

The authors assert that all procedures contributing to this work comply with the ethical standards of the relevant national and institutional committees on human experimentation and with the Helsinki Declaration of 1975 , as revised in 2008. The authors assert that all procedures contributing to this work comply with the ethical standards.

\section{References}

American Psychiatric Association (1987). Diagnostic and Statistical Manual of Mental Disorders (3rd edn. revised) (DSM-III-R). American Psychiatric Association:

Washington, DC.

American Psychiatric Association (2013). Diagnostic and Statistical Manual of Mental Disorders: DSM-5. American Psychiatric Association: Washington, DC.

Badcock JC, Dragovic M (2006). Schizotypal personality in mature adults. Personality and Individual Differences 40, 77-85.

Barrantes-Vidal N, Grant P, Kwapil T (2015). The role of schizotypy in the study of the etiology of schizophrenia spectrum disorders. Schizophrenia Bulletin 41, S408-S416.

Barrantes-Vidal N, Gross G, Sheinbaum T, Mitjavila M, Ballespí S, Kwapil TR (2013). Positive and negative schizotypy are associated with prodromal and schizophreniaspectrum symptoms. Schizophrenia Research 145, 50-55.

Bora E, Arabaci LE (2009). Confirmatory factor analysis of schizotypal personality traits in university students. Turkish Journal of Psychiatry 20, 339-345.

Brown TA (2015). Confirmatory Factor Analysis for Applied Research, 2nd edn. Guilford Press: New York.

Byrne B (2012). Structural Equation Modeling with Mplus: Basic Concepts, Applications, and Programming. Routledge, Taylor \& Francis Group.

Chen WJ, Hsiao CK, Lin CCH (1997). Schizotypy in community samples: the three-factor structure and correlation with sustained attention. Journal of Abnormal Psychology 106, 649-654.

Cheung MW-L, Leung K, Au K (2006). Evaluating multilevel models in cross-cultural research. Journal of Cross-Cultural Psychology 37, 522-541.

Chmielewski M, Watson D (2008). The heterogeneous structure of schizotypal personality disorder: item-level factors of the Schizotypal Personality Questionnaire and Their associations with obsessive-compulsive disorder symptoms, dissociative tendencies, and normal personality. Journal of Abnormal Psychology 117, 364-376.

Cicero DC (2016). Measurement Invariance of the Schizotypal Personality Questionnaire in Asian, Pacific Islander, White, and Multiethnic Populations. Psychological Assessment 28, 351-361.

Cicero DC, Martin EA, Becker TM, Docherty AR, Kerns JG (2014). Correspondence between psychometric and clinical high risk for psychosis in an undergraduate population. Psychological Assessment 26, 901-915.

Cohen A, Fonseca-Pedrero E (in press). Towards a schizotypy core: convergence and divergence of two 
empirically-derived self-report measures from a nonclinical sample. Journal of Experimental Psychopathology.

Cohen A, Mohr C, Ettinger U, Chan RCK, Park S (2015). Schizotypy as an organizing framework for social and affective sciences. Schizophrenia Bulletin 41, S427-S435.

Cohen AS, Matthews RA, Najolia GM, Brown LA (2010). Toward a more psychometrically sound brief measure of schizotypal traits: introducing the SPQ-Brief Revised. Journal of Personality Disorders 24, 516-537.

Cohen J (1988). Statistical Power Analysis for the Behavioral Sciences, 2nd edn. Lawrence Erlbaum Associates: Hillsdale, NJ.

Compton MT, Goulding SM, Bakeman R, McClure-Tone EB (2009). Confirmation of a four-factor structure of the Schizotypal Personality Questionnaire among undergraduate students. Schizophrenia Research 111, 46-52.

Debbané M, Eliez S, Badoud D, Conus P, Flückiger R, Schultze-Lutter F (2015). Developing psychosis and its risk states through the lens of schizotypy. Schizophrenia Bulletin 41, S396-S407.

Debbané M, Mohr C (2015). Integration and development in schizotypy research: an introduction to the special supplement. Schizophrenia Bulletin 41, S363-S365.

Dumas P, Bouafia S, Gutknecht C, Saoud M, Dalery J, d'Amato T (2000). [Validation of the French version of the Raine Schizotypal Personality Disorder Questionnaire categorial and dimensional approach to schizotypal personality traits in a normal student population]. Encephale 26, 23-29.

Dunn TJ, Baguley T, Brunsden V (2014). From alpha to omega: a practical solution to the pervasive problem of internal consistency estimation. British Journal of Psychology 105, 399-412.

Ettinger U, Mohr C, Gooding D, Cohen A, Rapp A, Haenschel C, Park S (2015). Cognition and brain function in schizotypy: a selective review. Schizophrenia Bulletin 41, S417-S426.

Everett KV, Linscott RJ (2015). Dimensionality vs taxonicity of schizotypy: some new data and challenges ahead. Schizophrenia Bulletin 41, S465-S474.

Fonseca Pedrero E, Debbané M (2017). Schizotypal traits and psychotic-like experiences during adolescence: an update. Psicothema 29, 5-17.

Fonseca-Pedrero E, Debbané M, Schneider M, Badoud D, Eliez S (2016a). Schizotypal traits in adolescents with 22q11.2 deletion syndrome: validity, reliability and risk for psychosis. Psychological Medicine 46, 1005-1013.

Fonseca-Pedrero E, Fumero A, Paino M, de Miguel A, Ortuño-Sierra J, Lemos Giraldez S, Muñiz J (2014). Schizotypal personality questionnaire: new sources of validity evidence in college students. Psychiatry Research 219, 214-220.

Fonseca-Pedrero E, Gooding D, Debbané M, Muñiz J (2016b). Psychopathology: psychosis assessment and high-risk paradigms. In The ITC International Handbook of Testing and Assessment (ed. D. Bartram), pp. 147-170. Oxford University Press: UK.

Fonseca-Pedrero E, Lemos-Giráldez S, Paino M, Villazón-García U, Muñiz J (2009). Validation of the schizotypal personality questionnaire brief form in adolescents. Schizophrenia Research 111, 53-60.

Fonseca-Pedrero E, Ortuño-Sierra J, Sierro G, Daniel C, Cella M, Preti A, Mohr C, Mason OJ (2015). The measurement invariance of schizotypy in Europe. European Psychiatry 30, 837-844.

Fossati A, Raine A, Carretta I, Leonardi B, Maffei C (2003). The three-factor model of schizotypal personality: invariance across age and gender. Personality and Individual Differences 35, 1007-1019.

Fusar-Poli P, Carpenter WT, Woods SW, McGlashan TH (2014). Attenuated psychosis syndrome: ready for DSM-5.1? Annual Review of Clinical Psychology 10, 155-192.

Gross GM, Mellin J, Silvia PJ, Barrantes-Vidal N, Kwapil TR (2014). Comparing the factor structure of the Wisconsin Schizotypy Scales and the Schizotypal Personality Questionnaire. Personality Disorders: Theory, Research, and Treatment 5, 397-405.

Hu L-T, Bentler PM (1999). Cut off criteria for fit indexes in covariance structure analysis: conventional criteria versus new alternatives. Structural Equation Modeling 6, 1-55.

IBM Corp Released (2013). IBM SPSS Statistics for Windows, Version 22.0. IBM Corp: Armonk, NY.

Kelleher I, Cederlöf M, Lichtenstein P (2014). Psychotic experiences as a predictor of the natural course of suicidal ideation: a Swedish cohort study. World Psychiatry 13, 184188.

Kendler KS, McGuire M, Gruenberg AM, O'Hare A, Spellman M, Walsh D (1993). The Roscommon Family Study: III. Schizophrenia-related personality disorders in relatives. Archives of General Psychiatry 50, 781-788.

Kendler KS, Myers J, Torgersen S, Neale MC, Reichborn-Kjennerud T (2007). The heritability of cluster A personality disorders assessed by both personal interview and questionnaire. Psychological Medicine 37, 655-665.

Kwapil TR, Barrantes-Vidal N (2015). Schizotypy: looking back and moving forward. Schizophrenia Bulletin 41, S366-S373.

Kwapil TR, Gross GM, Silvia PJ, Barrantes-Vidal N (2013). Prediction of psychopathology and functional impairment by positive and negative schizotypy in the Chapmans' ten-year longitudinal study. Journal of Abnormal Psychology 122, 807-815.

Lahmar ML, Gassab L, Beltaief F, Mechri A (2014). Psychometric properties of the Arabic version of the schizotypal personality questionnaire in Tunisian university students. Tunisie Medicale 92, 318-322.

Lenzenweger MF (2010). Schizotypy and Schizophrenia: The View from Experimental Psychopathology. Guilford Press: New York.

Liddle PF (1987). The symptoms of chronic schizophrenia. A re-examination of the positive-negative dichotomy. British Journal of Psychiatry 151, 145-151.

Linscott RJ (2013). The taxonicity of schizotypy: does the same taxonic class structure emerge from analyses of different attributes of schizotypy and from fundamentally different statistical methods? Psychiatry Research 210, 414-421.

Linscott RJ, van Os J (2013). An updated and conservative systematic review and meta-analysis of epidemiological 
evidence on psychotic experiences in children and adults: on the pathway from proneness to persistence to dimensional expression across mental disorders. Psychological Medicine 43, 1133-1149.

Mason O (2015). The assessment of schizotypy and its clinical relevance. Schizophrenia Bulletin 41, S374-S385.

Meehl PE (1962). Schizotaxia, schizotypy, schizophrenia. American Psychologist 17, 827-838.

Muthén LK, Muthén BO (1998-2012). Mplus User's Guide, 7th edn. Muthén \& Muthén: Los Angeles, CA.

Nuevo R, Chatterji S, Verdes E, Naidoo N, Arango C, Ayuso-Mateos JL (2012). The continuum of psychotic symptoms in the general population: a cross-national study. Schizophrenia Bulletin 38, 475-485.

R Development Core Team (2011). R: A Language and Environment for Statistical Computing. R Foundation for Statistical Computing (http://www.R-project.org): Vienna, Austria.

Raine A (1991). The SPQ: a scale for the assessment of schizotypal personality based on DSM-III-R criteria. Schizophrenia Bulletin 17, 555-564.

Raine A (2006). Schizotypal personality: neurodevelopmental and psychosocial trajectories. Annual Review of Clinical Psychology 2, 291-326.

Raine A, Benishay D (1995). The SPQ-B: a brief screening instrument for schizotypal personality disorder. Journal of Personality Disorders 9, 346-355.

Raine A, Reynolds C, Lencz T, Scerbo A, Triphon N, Kim D (1994). Cognitive-perceptual, interpersonal, and disorganized features of schizotypal personality . Schizophrenia Bulletin 20, 191-201.

Reynolds CA, Raine A, Mellingen K, Venables PH, Mednick SA (2000). Three-factor model of schizotypal personality: invariance across culture, gender, religious affiliation, family adversity, and psychopathology. Schizophrenia Bulletin 26, 603-618.
Schimanski ID, Mouat KL, Billinghurst BL, Linscott RJ (2017). Preliminary evidence that schizophrenia liability at age 15 predicts suicidal ideation two years later. Schizophrenia Research 181, 60-62.

Stefanis NC, Smyrnis N, Avramopoulos D, Evdokimidis I, Ntzoufras I, Stefanis CN (2004). Factorial composition of self-rated schizotypal traits among young males undergoing military training. Schizophrenia Bulletin 30, 335-350.

Tsaousis I, Zouraraki C, Karamaouna P, Karagiannopoulou L, Giakoumaki SG (2015). The validity of the Schizotypal Personality Questionnaire in a Greek sample: tests of measurement invariance and latent mean differences. Comprehensive Psychiatry 62, 51-62.

Vollema MG, Hoijtink H (2000). The multidimensionality of self-report schizotypy in a psychiatric population: an analysis using multidimensional Rasch models. Schizophrenia Bulletin 26, 565-575.

Walter EE, Fernandez F, Snelling M, Barkus E (2016). Genetic consideration of schizotypal traits: a review. Frontiers in Psychology 7, 1769.

World Medical Association (2013). World Medical Association Declaration of Helsinki: ethical principles for medical research involving human subjects. Journal of the American Medical Association 310, 2191-2194.

Wuthrich VM, Bates TC (2006). Confirmatory factor analysis of the three-factor structure of the Schizotypal Personality Questionnaire and Chapman schizotypy scales. Journal of Personality Assessment 87, 292-304.

Yu CY, Muthén BO (2002). Evaluation of Model Fit Indices for Latent Variable Models with Categorical and Continuous Outcomes (Technical Report). UCLA, Graduate School of Education and Information Studies: Los Angeles.

Zumbo BD, Gadermann AM, Zeisser C (2007). Ordinal versions of coefficients alpha and theta for Likert rating scales. Journal of Modern Applied Statistical Methods 6, $21-29$. 